\title{
Role of Present Life Style in Diseases
}

\section{Jayesh V Trivedi*}

Gujarat Adani Institute of Medical Science, India

Submission: December 15, 2017; Published: January 17, 2017

${ }^{*}$ Corresponding author: Jayesh V Trivedi, Head Of Department And Professor Of Medicine, Gujarat Adani Institute of Medical Science, Bhuj Gujarat, India, Email: drjvtrivedi@rediffmail.com

\section{Introduction}

Good days are coming like end of 2016, everybody is in light mood to enjoy new coming year. At the outset I would like to wish you all the best for New Year. Let me talk something to you regarding the anticipated medical problems which our next generations are likely to face.

1. The incidence of Type 2 Diabetes at a younger age is growing up very fast. Apart from genetic predisposition the present culture and lifestyle is responsible. We eat high carbohydrate riched diet with saturated fatty acids and transfatty acids. Junk food is equally responsible as it contains less fiber content and having high calories. Young people do not undergo heavy brisk exercise for $40 \mathrm{~min}$ a day for 7 days a week. Which results in to insulin resistance and fatty infiltration of liver resulting in to Non-infective Hepatitis and Cirrhosis of liver?

2. Use of tobacco and its products play a big role in early onset of Hypertension, Dyslipidemia, and Coronary Arterial Disease at a young age and Strokes. Consumption of Alcohol has also increased which increases Hypertension. Salt consumption has also increased in recent days.

3. These days HDL Cholesterol is invariably low and Non HDL Cholesterol is very high. Diet is having more concentration of Omega 6 fatty acids and less Omega 3 fatty acids. This results in to alteration of their normal ratio of 1:1 to 20:1, which is responsible for truncal obesity, Type2 Diabetes Mellitus, polycysticovariandisease, Dyslipidemia, Hyperuricemia and Early Coronary Artery Disease

Presently life has become very competitive and young people are ambitious. So from school days only they are facing lots of stress and strain. The relaxation exercise, preksha dhyan or mediation measures are lacking in present life style which increases the level of stress in them. Young people needs to do serving as an to keep their baro reflexes intake.

I wish Santa will give us this gift to our new generation and make them physically and mentally strong to face the upcoming challenges of life.
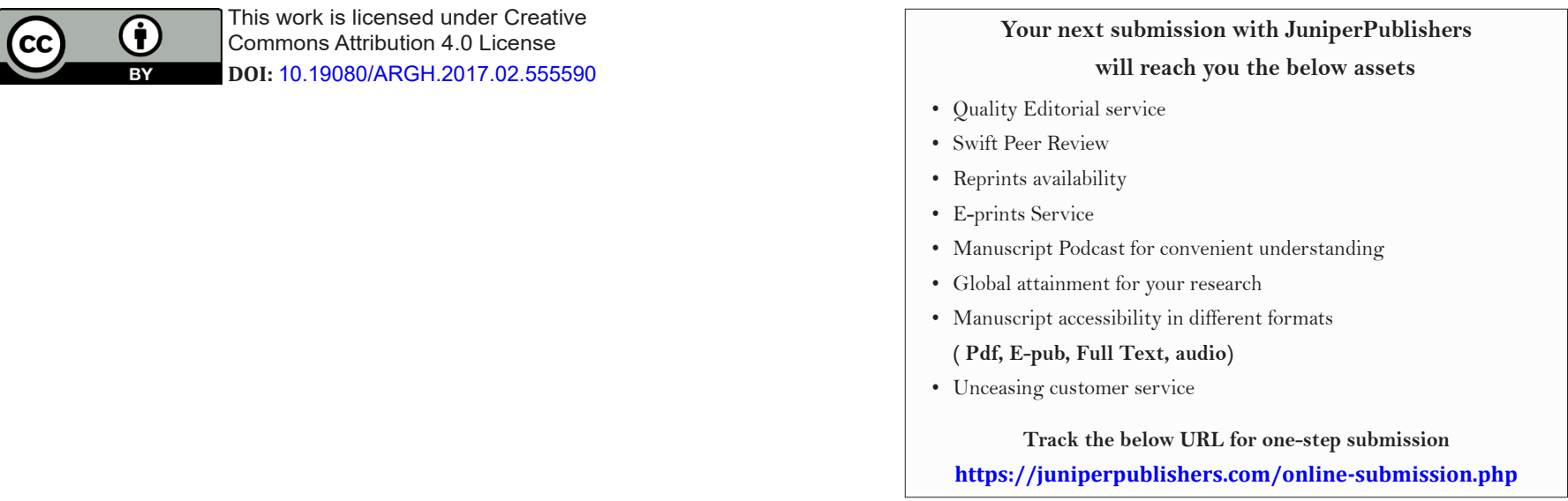